\title{
Ensino de Design de IHC: Um Panorama sobre a Análise e Modelagem de Usuários, Tarefas e Interação
}

\author{
Bruna Camila de Menezes ${ }^{1}$, Leonardo Cunha de Miranda ${ }^{1}$ \\ ${ }^{1}$ Departamento de Informática e Matemática Aplicada \\ Universidade Federal do Rio Grande do Norte (UFRN) \\ 59078-970 - Natal, RN - Brasil \\ brunacamilamenezes@gmail.com, leonardo@dimap.ufrn.br
}

\begin{abstract}
Resumo. Há muitos anos os pesquisadores brasileiros da área de Interação Humano-Computador (IHC) vêm discutindo o Ensino de IHC no país. Este artigo visa a contribuir com as discussões acerca do ensino de design de IHC ao apresentar um estudo exploratório realizado com o intuito de identificar se conteúdos relativos a análise e modelagem de usuários, tarefas e interação estão sendo ensinados em disciplinas da área, e quais são os conteúdos que estão sendo abordados relacionados a esses tópicos.
\end{abstract}

\section{Introdução}

Nas últimas décadas a comunidade brasileira de Interação Humano-Computador (IHC) vem discutindo o Ensino de IHC nos cursos de Computação do Brasil e, mais sistematicamente, a partir da criação, em 2006, de um Grupo de Trabalho (GT) durante a realização do VII Simpósio Brasileiro sobre Fatores Humanos em Sistemas Computacionais. Resultados deste GT foram apresentados por Silveira e Prates (2007) e, posteriormente, revisados por Bim et al. (2012). Na ementa proposta pelo GT estão descritos tópicos variados que tratam do design de IHC e que incluem a análise e modelagem de usuários, tarefas e interação. Os conteúdos relativos a esses tópicos são: elicitação e análise, modelagem de tarefas, e modelagem de interação. Cabe notar que a ementa não detalhada o que deve ser ensinado em relação a cada um desses três tópicos.

Nesse contexto, o objetivo deste trabalho é identificar, através de um estudo exploratório, se os tópicos supradescritos estão sendo explorados em disciplinas relacionadas à área de IHC. Esses foram os tópicos escolhidos para serem investigados neste estudo, visto sua relevância no projeto de interface de usuário. Assim, as questões de pesquisa deste trabalho são: (i) Disciplinas relacionadas à área de IHC estão explorando conteúdos relativos à análise e modelagem de usuários, tarefas e interação? (ii) Quais são os conteúdos relativos a estes três tópicos que estão sendo ensinados? (iii) Os professores que lecionam disciplinas relacionadas à área de IHC também são autores de artigos no principal Simpósio da área do Brasil?

Este artigo está organizado da seguinte maneira: a Seção 2 apresenta a fundamentação; a Seção 3 descreve o método; a Seção 4 apresenta os resultados; a Seção 5 discute os resultados; e a Seção 6 conclui o artigo.

\section{Fundamentação}

Durante o projeto de interface de usuário tem-se frequentemente utilizado modelos, por eles permitirem uma representação de informações de design com alto grau de 
abstração, além do uso de modelos também permitir reflexões sobre decisões alternativas de design [Winckler e Pimenta 2004]. Segundo Souza et al. (1999), ao se projetar a interface de um sistema interativo, deve-se ter uma atenção especial aos usuários que irão utilizá-la. Para isso, pode-se utilizar a análise e modelagem de usuário.

A realização da análise e modelagem de usuários contribui para a melhor caracterização dos usuários, possibilitando especificar, por exemplo, quais funções são exercidas, e quais atividades o usuário pode realizar. Leite (2010) explica que a análise de usuário possibilita o conhecimento dos papéis dos usuários pelos desenvolvedores de sistemas, e esse conhecimento faz com que os desenvolvedores compreendam mais seus stakeholders, podendo prevenir possíveis erros de interação, e melhorando aplicações em desenvolvimento. Já Souza et al. (1999) destacam alguns fatores para a realização da análise de usuário, que são: papel ou função do usuário (que é definido pelas tarefas que o usuário realiza), familiaridade com computadores, familiaridade com a aplicação, frequência da uso da aplicação, e contexto sociocultural. Exemplos de conteúdos a serem ensinados: perfil de usuário (feito a partir da coleta de dados dos usuários), personas, e cenários de uso.

Em relação a análise e modelo de tarefas, Winckler e Pimenta (2004) destacam que a análise de tarefas teve sua origem em métodos empíricos vindos da Ergonomia. Apesar de sua origem ser na Ergonomia, logo se percebeu a utilidade do uso da análise de tarefas em outras áreas, como na Computação. Segundo Muñoz (2000), o processo de análise de tarefas consiste em um método iterativo de coleta de dados, em que são obtidas as metas do usuário e as tarefas que serão realizadas para que seja alcançado seu objetivo. A análise de tarefas busca fornecer ao designer uma visão clara sobre como os usuários realizam tarefas e de que modo eles poderiam realizá-las via um sistema interativo. Já os modelos de tarefa são utilizados para prover uma melhor visualização da realização de tarefas, e a partir desses modelos pode-se refletir sobre como tornar os procedimentos mais eficientes, melhorando o design de sistemas. Os modelos de tarefas também são utilizados para formalizar os resultados extraídos na análise de tarefas, enfatizando alguma perspectiva escolhida [Winckler e Pimenta 2004]. Para Paternó et al. (1997) o modelo de tarefas é "uma descrição lógica das atividades a serem executadas para alcançar os objetivos do usuário". Exemplos de modelos a serem ensinados: CTT (ConcurTaskTrees) [Paternó et al. 1997], HTA (Hierarchical Tasks Analysis) [Annett 2003], e GOMS (Goals, Operators, Methods, Selection Rules) e suas variações, tal como, KLM (Keystroke Level Model) [Card et al. 1983].

Com os resultados obtidos através da análise e da modelagem de usuários e de tarefas, temos informações suficientes para pensar como serão dadas as interações dos usuários com o sistema. Para isso, utilizamos a modelagem de interação que consiste em projetar todos as possíveis formas de interação que o usuário pode escolher para alcançar um objetivo. Segundo Costa Neto e Leite (2011), a modelagem de interação possibilita a visão da interação e navegação pela interface projetada, sem que haja uma definição formal do layout da aplicação. Isso faz com que o foco do designer esteja no processo de interação e não em detalhes expressos pela organização da interface. Para Silveira e Prates (2007), a modelagem de interação tem por objetivo definir as conversas que o usuário deve travar com o designer para que seja formulada as interações do usuário com o sistema. Exemplos de conteúdos a serem ensinados: TAG (Task-Action Grammar) [Payne e Green 1989], MoLIC (Modeling Language for Interaction as Conversation) [Lopes et al. 2015], e UAN (User Action Notation) [Hix e Hartson 1993]. 


\section{Método}

Numa primeira etapa, foram realizadas buscas na Internet com o intuito de obter slides de aulas de disciplinas relacionadas à área de IHC. Após essa coleta, considerando o objetivo deste trabalho, conforme descrito na Seção 1, foram analisados os slides encontrados com o intuito de coletar dados acerca do ensino dos conteúdos de interesse deste estudo. Após essa etapa, foi realizada uma busca no catálogo de artigos do Simpósio Brasileiro sobre Fatores Humanos em Sistemas Computacionais (IHC-Brasil), com o intuito de identificar se os professores que lecionaram as disciplinas identificadas na primeira etapa deste estudo são, ou não, autores de artigos publicados no IHC-Brasil.

\section{Resultados}

Foram identificadas 31 disciplinas relacionadas à área de IHC que foram lecionadas por 25 professores que atuam em 19 Instituições de Ensino (IEs). A Tabela 1 apresenta uma síntese dos dados levantados a partir da análise dos slides que foram coletados. Cabe ressaltar que neste trabalho identificamos os professores e as IEs, respectivamente, por P $x$ e IEx, e as células com "n.d." significa que a informação estava indisponível. Ainda, os níveis do ensino das disciplinas foram abreviados: Graduação (G), Especialização (E) e Pós-graduação (PG).

Tabela 1. Síntese dos dados coletados.

\begin{tabular}{|c|c|c|c|}
\hline $\begin{array}{c}\text { Px } \\
(\text { IEx } x) \\
\end{array}$ & $\begin{array}{c}\text { Nome da disciplina } \\
\text { (Nível do ensino, Ano de oferta) } \\
\end{array}$ & Título da aula & Conteúdos explorados de interesse deste estudo \\
\hline \multirow{9}{*}{$\begin{array}{c}\text { P1 } \\
\text { (IE1) }\end{array}$} & \multirow{9}{*}{$\begin{array}{l}\text { Introdução a IHC } \\
\quad(\mathrm{G}, 2013)\end{array}$} & Projeto de IHC & Conceitos de modelagem de usuário \\
\hline & & Identificação de necessidades dos usuários e requisitos de IHC & Análise e modelagem de usuário: Perfil de usuário \\
\hline & & Design de IHC - Organização do espaço de problema & $\begin{array}{l}\text { Personas e cenários; modelo e análise de tarefas: HTA, } \\
\text { GOMS e CTT }\end{array}$ \\
\hline & & Design de IHC - Design da comunicação modelos de interação & Conceitos de modelagem de usuário \\
\hline & & $\begin{array}{l}\text { Cenários de projeto e levantamento das necessidades dos usuários } \\
\text { II }\end{array}$ & Modelagem de interação (MoLIC); HTA \\
\hline & & \begin{tabular}{|l|} 
Design de IHC - Revisão modelo de tarefas e projeto de curso \\
\end{tabular} & HTA \\
\hline & & Revisão para P2 & Modelos de tarefa; modelagem de interação; cenários \\
\hline & & Modelo de tarefas & Modelo e análise de tarefas: GOMS, HTA e CTT \\
\hline & & $\begin{array}{l}\text { Modelo de interação (revisão) Design de IHC da interação para o } \\
\text { design da interface }\end{array}$ & Modelo de Interação - MoLIC \\
\hline \multirow{6}{*}{$\begin{array}{c}\text { P2 } \\
(\mathrm{IE} 1)\end{array}$} & $\begin{array}{c}\text { Introdução a Interação Humano- } \\
\text { Computador (IHC) } \\
(\mathrm{G}, 2011) \\
\end{array}$ & Análise e modelagem de tarefas & HTA, CTT, GOMS e KLM \\
\hline & \multirow{5}{*}{$\begin{array}{l}\text { Introdução a IHC } \\
\quad(\mathrm{G}, 2014)\end{array}$} & Avaliação em IHC: Hierarquia de metas e testes com usuários & HTA \\
\hline & & Design de IHC - Organização do espaço de problema & $\begin{array}{l}\text { Personas e cenários; modelo e análise de tarefas: HTA, } \\
\text { GOMS e CTT }\end{array}$ \\
\hline & & \begin{tabular}{|l|} 
MoLIC - Modelagem da interação \\
\end{tabular} & MoLIC \\
\hline & & \begin{tabular}{|l|} 
MoLIC - Modelagem da interação 2 \\
\end{tabular} & MoLIC \\
\hline & & Relembrando: Cenário de problema & $\begin{array}{l}\text { Análise e modelagem de tarefas: HTA, CTT, GOMS e } \\
\text { KLM }\end{array}$ \\
\hline \multirow{6}{*}{$\begin{array}{l}\text { P3 } \\
(\text { IE1) }\end{array}$} & \multirow{2}{*}{$\begin{array}{l}\text { Introdução a IHC } \\
\quad(\mathrm{G}, 2011)\end{array}$} & Projeto de IHC - Modelos de interação com MoLIC & MoLIC \\
\hline & & Projeto de IHC Modelos de tarefa e interação & $\begin{array}{l}\text { Modelagem de tarefas GOMS, modelagem de interação } \\
\text { MoLIC }\end{array}$ \\
\hline & \multirow{3}{*}{$\begin{array}{l}\text { Introdução a IHC } \\
\qquad(\mathrm{G}, 2014)\end{array}$} & Projeto de Curso Uso de cenários em projeto & Modelagem de tarefas e interação, e perfil de usuário \\
\hline & & $\begin{array}{l}\text { Projeto de curso Levantamento de necessidades dos usuários } \\
\text { entrevistas }\end{array}$ & $\begin{array}{l}\text { Entrevista, e perfil de usuário: análise/modelagem de } \\
\text { usuário }\end{array}$ \\
\hline & & Modelagem de tarefas e interação & Modelagem de tarefas e interação: GOMS e MoLIC \\
\hline & $\begin{array}{l}\text { Introdução a IHC } \\
\text { (PG, 2014) }\end{array}$ & Métodos e modelos formais em IHC & Modelo de tarefa \\
\hline \multirow{5}{*}{$\begin{array}{c}\text { P4 } \\
(\mathrm{IE} 2)\end{array}$} & \multirow{4}{*}{$\begin{array}{c}\text { Introdução à Interação Humano- } \\
\text { Computador } \\
(\mathrm{G}, 2006)\end{array}$} & Análise e modelagem de usuário & Análise e modelagem de usuário, e perfil de usuário \\
\hline & & Análise de tarefas & Análise e modelagem de tarefas \\
\hline & & \begin{tabular}{|l|l} 
Modelagem de tarefas \\
\end{tabular} & GOMS, KLM e HTA \\
\hline & & Modelagem de interação & TAG, MoLIC e UAN \\
\hline & $\begin{array}{l}\text { Introdução à Interação Humano- } \\
\text { Computador } \\
\text { (n.d.) }\end{array}$ & Modelos e técnicas de modelagem em IHC & $\begin{array}{l}\text { Análise e modelagem de tarefas: TAG, UAN, GOMS e } \\
\text { KLM; cenários, análise e modelagem de usuário }\end{array}$ \\
\hline \multirow{2}{*}{$\begin{array}{c}\text { P5 } \\
\text { (IE3) }\end{array}$} & $\begin{array}{l}\text { Interação Humano-Computador } \\
(\mathrm{G}, 2016)\end{array}$ & $\begin{array}{l}\text { Interação Humano-Computador (IHC) Avaliação: usabilidade e } \\
\text { acessibilidade }\end{array}$ & $\begin{array}{l}\text { Análise e modelagem de tarefas (GOMS) e análise de } \\
\text { usuários, e cenários }\end{array}$ \\
\hline & $\begin{array}{l}\text { Interação Humano-Computador } \\
(\mathrm{G}, 2016)\end{array}$ & Modelos de Sistemas cap 05 livro engenharia de software & Modelagem de interação \\
\hline $\begin{array}{c}\mathrm{P} 6 \\
\text { (IE3) }\end{array}$ & $\begin{array}{c}\text { Interação humano computador (IHC) } \\
(\mathrm{G}, 2017)\end{array}$ & \multicolumn{2}{|c|}{ Nenhuma aula/conteúdo relacionado ao escopo deste estudo } \\
\hline \multirow{6}{*}{$\begin{array}{l}\text { P7 } \\
\text { (IE4) }\end{array}$} & \multirow{6}{*}{$\begin{array}{l}\text { Interface Homem/Máquina } \\
\qquad(\mathrm{G}, 2011)\end{array}$} & Interface Homem/Máquina - Aula 10 & Personas \\
\hline & & Interface Homem/Máquina - Aula 11 & HTA, GOMS, CTT e KLM \\
\hline & & Interface Homem/Máquina - Aula 14 & Cenários de interação \\
\hline & & Design de IHC & Modelagem de tarefa e de interação: MoLIC \\
\hline & & \begin{tabular}{|l} 
Design da Interface (Parte I) \\
\end{tabular} & Design de interface \\
\hline & & Interface Homem/Máquina - Aula 23 & Coleta de dados de interação \\
\hline
\end{tabular}




\begin{tabular}{|c|c|c|c|}
\hline & & Interface Homem/Máquina - Aula 25 & Coleta de dados interpretação dos resultados \\
\hline \multirow{2}{*}{$\begin{array}{l}\text { P8 } \\
\text { (IE4) }\end{array}$} & \multirow{2}{*}{$\begin{array}{l}\text { Interação Humano-Computador } \\
\qquad(\mathrm{G}, 2012)\end{array}$} & $\begin{array}{l}\text { Interação Humano-Computador Projeto de Interface (Análise do } \\
\text { Usuário e da Tarefa) }\end{array}$ & $\begin{array}{l}\text { Análise e modelagem da tarefa e do usuário, cenários; } \\
\text { GOMS }\end{array}$ \\
\hline & & Interação Humano-Computador Surveys & Modelagem de usuário \\
\hline $\begin{array}{l}\text { P9 } \\
\text { (IE5) }\end{array}$ & \begin{tabular}{|c|} 
Interface Homem Computador (IHC) \\
(n.d., n.d.)
\end{tabular} & Nenhuma aula/conteúdo relaciona & ado ao escopo deste estudo \\
\hline \multirow{3}{*}{$\begin{array}{l}\text { P10 } \\
\text { (IE6) }\end{array}$} & \multirow{3}{*}{$\begin{array}{c}\text { - Engenharia de Usabilidade } \\
(\mathrm{G}, 2004)\end{array}$} & Engenharia de Usabilidade: Um a abordagem Ergonômica & Análise e modelagem de usuário \\
\hline & & AHT - Análise hierárquica da tarefa & HTA \\
\hline & & \begin{tabular}{|l} 
Avaliação de usabilidade de IHC \\
\end{tabular} & CTT \\
\hline $\begin{array}{c}\text { P11 } \\
\text { (IE6) } \\
\end{array}$ & $\begin{array}{c}\text { Interação Humano-Computador } \\
(\mathrm{G}, 2003)\end{array}$ & Avaliação de interfaces: Conceitos e métodos & Perfil de usuário, análise preditiva (menciona modelos) \\
\hline \multirow{2}{*}{$\begin{array}{c}\text { P12 } \\
\text { (IE7) }\end{array}$} & \multirow{2}{*}{$\begin{array}{l}\text { Interação Humano-Computador } \\
\text { (n.d., 2015) }\end{array}$} & Interação Humano-Computador - Processo de design & Design de interação; personas; Análise de tarefas: HTA \\
\hline & & Interação Humano-Computador - Prototipagem & Storyboard \\
\hline \multirow{3}{*}{$\begin{array}{l}\text { P13 } \\
\text { (IE7) }\end{array}$} & \multirow{3}{*}{$\begin{array}{l}\text { Interação Humano-Computador } \\
\text { (n.d., 2013) }\end{array}$} & Abordagens teóricas & Modelo de interação \\
\hline & & \begin{tabular}{|l} 
Processos de design de IHC (Parte I) \\
\end{tabular} & Menciona análise de tarefas e de usuário \\
\hline & & Design de IHC & $\begin{array}{l}\text { Modelo de tarefa: HTA; modelagem de interação: } \\
\text { MoLIC }\end{array}$ \\
\hline \multirow{2}{*}{\begin{tabular}{c|c|} 
P14 \\
(IE8)
\end{tabular}} & \multirow{2}{*}{$\begin{array}{c}\text { n.d. } \\
(\mathrm{G}, 2013) \\
\end{array}$} & IHC II & Menciona modelo de usuário \\
\hline & & Usabilidade II & Análise de tarefas: GOMS; cenários \\
\hline \multirow{2}{*}{$\begin{array}{l}\text { P15 } \\
\text { (IE9) } \\
\end{array}$} & \multirow{2}{*}{$\begin{array}{c}\text { Interação Humano-Computador } \\
(\mathrm{G}, \text { n.d.) }\end{array}$} & Aula 06 & Fala um pouco sobre modelo de usuário \\
\hline & & Aula 10 & Perfil de usuário - Análise e modelagem de usuário \\
\hline \multirow{2}{*}{$\begin{array}{c}\mathrm{P} 16 \\
(\mathrm{IE} 10) \\
\end{array}$} & \multirow{2}{*}{$\begin{array}{l}\text { Interação Humano-Computador } \\
(\mathrm{G}, 2016)\end{array}$} & Como coletar dados dos usuários? & Perfil de usuário - Análise e modelagem de usuário \\
\hline & & Espaço problema & Persona; cenários; análise de tarefa: HTA e GOMS \\
\hline \multirow{2}{*}{$\begin{array}{c}\text { P17 } \\
\text { (IE11) } \\
\end{array}$} & \multirow{2}{*}{$\begin{array}{l}\text { Interface Homem Máquina } \\
(\mathrm{G}, 2014)\end{array}$} & Interface Homem Máquina - Aula 1 & Análise de tarefas \\
\hline & & \begin{tabular}{|l|} 
Análise da tarefa \\
\end{tabular} & HTA \\
\hline \multirow{2}{*}{$\begin{array}{c}\text { P18 } \\
\text { (IE12) }\end{array}$} & \multirow{2}{*}{$\begin{array}{c}\text { Interação Humano- Computador: uma } \\
\text { ciência de design } \\
(\mathrm{G}, 2015)\end{array}$} & O modelo GOMS & GOMS \\
\hline & & Meta-modelos de processos de design de interfaces de usuário & $\begin{array}{l}\text { Menciona análise de tarefas e fala sobre modelagem de } \\
\text { usuário; design da interação }\end{array}$ \\
\hline \multirow{3}{*}{$\begin{array}{c}\text { P19 } \\
\text { (IE13) }\end{array}$} & \multirow{3}{*}{$\begin{array}{l}\text { Interface Homem Computador (IHC) } \\
\qquad(\mathrm{G}, 2016)\end{array}$} & Introdução & Menciona análise de usuário, e análise da tarefa: HTA \\
\hline & & Requisitos e análise do usuário & $\begin{array}{l}\text { Análise de usuário; análise de tarefa: GOMS, UAN, TAG } \\
\text { e HTA; cenários }\end{array}$ \\
\hline & & Projeto de interação com o usuário: Avaliação de interação & Projeto de usuário \\
\hline $\begin{array}{l}\text { P20 } \\
\text { (IE14) }\end{array}$ & $\begin{array}{l}\text { Interação Humano-Computador } \\
\text { Introdução } \\
(\mathrm{G}, 2017) \\
\end{array}$ & $\begin{array}{l}\text { Organização do espaço do problema para design de IHC: } \\
\text { Modelagem de usuários, tarefas e interação }\end{array}$ & $\begin{array}{l}\text { Modelos de usuários: Perfil de usuário e personas; } \\
\text { modelos de tarefas: GOMS, HTA e CTT; modelos de } \\
\text { interação: MoLIC }\end{array}$ \\
\hline \multirow{3}{*}{$\begin{array}{c}\text { P21 } \\
\text { (IE15) }\end{array}$} & Design e usabilidade em IHC & Teoria para IHC: Engenharia Semiótica & Menciona análise de usuário e de tarefas \\
\hline & (n.d., 2010) & $\mathrm{O}$ design de IHC & Análise de usuário \\
\hline & $\begin{array}{c}\text { n.d. } \\
\text { (n.d, 2003) } \\
\end{array}$ & Análise de usuário e tarefas & $\begin{array}{l}\text { Análise e modelagem de tarefas: GOMS; análise de } \\
\text { usuário; uso de cenários }\end{array}$ \\
\hline $\begin{array}{c}\text { P23 } \\
\text { (IE17) } \\
\end{array}$ & $\begin{array}{c}\text { Software Educacional I } \\
(\mathrm{G}, 2003)\end{array}$ & Interface Homem-Computador (IHC) & Modelagem de usuário \\
\hline $\begin{array}{c}\text { P24 } \\
\text { (IE18) } \\
\end{array}$ & $\begin{array}{c}\text { Interação Humano-Computador (IHC) } \\
(\mathrm{G}, 2014)\end{array}$ & \multicolumn{2}{|c|}{ Nenhuma aula/conteúdo relacionado ao escopo deste estudo } \\
\hline $\begin{array}{l}\text { P25 } \\
\text { (IE19) }\end{array}$ & $\begin{array}{c}\text { Projeto e Avaliação de Interfaces: } \\
\text { Ambiente Web } \\
\text { (E, 2009) }\end{array}$ & \multicolumn{2}{|c|}{ Nenhuma aula/conteúdo relacionado ao escopo deste estudo } \\
\hline
\end{tabular}

De forma geral, identificamos que 27 disciplinas $(87,1 \%)$ oferecem conteúdos para explorar ao menos um dos tópicos de design de IHC de interesse deste estudo, ou seja, apenas 4 disciplinas (12,9\%) não exploraram nenhum dos três tópicos. Também foi constatado que 22 disciplinas (71\%) não apresentam conteúdos para cobrir, simultaneamente, os três tópicos investigados neste estudo, ou seja, apenas 9 disciplinas (29\%) exploram os três tópicos. Especificamente, em relação à análise e modelagem de usuários, 11 disciplinas $(35,5 \%)$ não exploram nenhum conteúdo sobre esse assunto (Figura 1a), e o conteúdo mais ensinado é cenários, seguido por perfil de usuário e personas (Figura 2a). No que diz respeito à análise e modelo de tarefas, 8 disciplinas $(25,8 \%)$ não exploram nenhum conteúdo sobre esse assunto (Figura 1b), e os modelos mais ensinados são GOMS, seguidos por HTA, KLM e CTT (Figura 2b). Já no que concerne a modelagem de interação, 18 disciplinas $(58,1 \%)$ não apresentam nenhum conteúdo sobre esse assunto (Figura 1c), e o conteúdo mais ensinado é o MoLIC, seguido por UAN e TAG (Figura 2c).
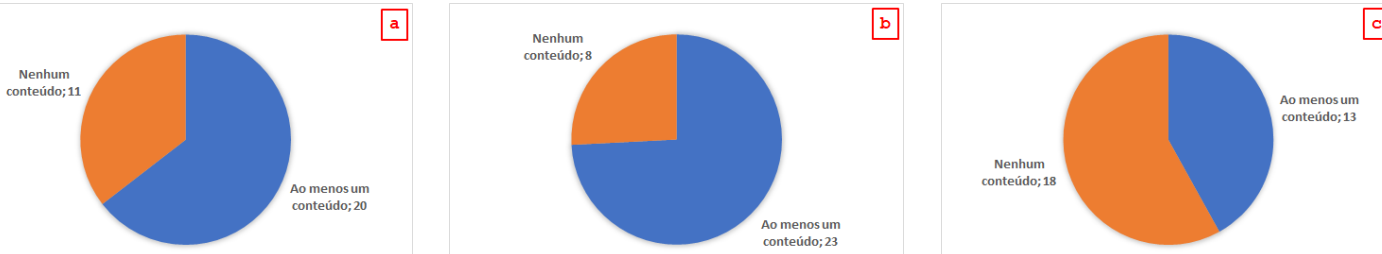

Figura 1. Conteúdos em disciplinas sobre (a) análise e modelagem de usuários, (b) análise e modelo de tarefas, e (c) modelagem de interação. 


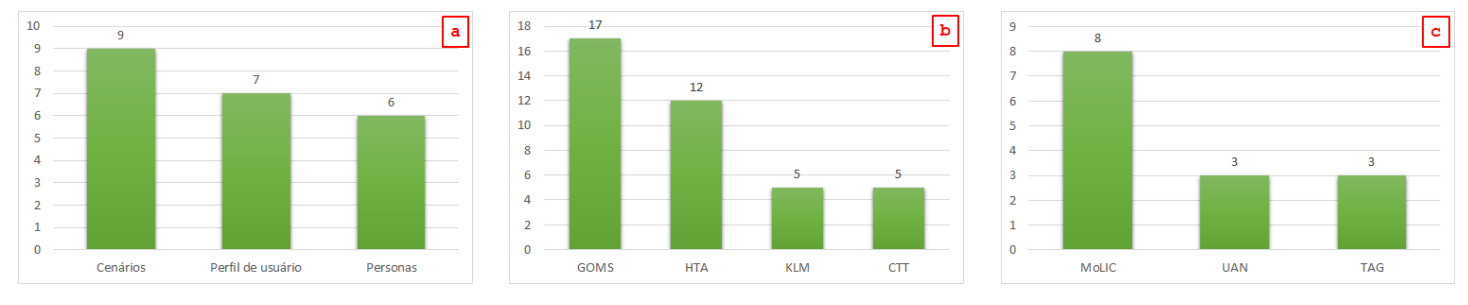

Figura 2. Conteúdos ensinados sobre (a) análise e modelagem de usuários, (b) análise e modelo de tarefas, e (c) modelagem de interação.

Apenas seis professores (24\%) exploram os três tópicos. Dos 15 professores $(60 \%)$ que nunca publicaram no IHC-Brasil, dois destes exploram os três tópicos. Assim, apenas $13 \%$ dos professores que nunca publicaram no IHC-Brasil exploram os três tópicos. Já dos 10 professores $(40 \%)$ que já publicaram ao menos um artigo no IHC-Brasil, quatro destes exploram os três tópicos. Assim, $40 \%$ dos professores que já publicaram ao menos um artigo no IHC-Brasil exploram os três tópicos. Também foi observado que os professores que exploram os três tópicos pertencem ou tem alguma ligação com uma IE específica e, também, são pesquisadores ativos na comunidade brasileira de IHC; alguns desses professores fizeram parte do GT descrito na Seção 1.

\section{Discussão}

Sabemos que cada IE e cada curso nas diferentes regiões do Brasil tem suas particularidades, então investigar o contexto de cada disciplina é importante para entender por que certos conteúdos são ou não explorados nas disciplinas. Dificilmente os slides de aula encontrados exploram os três tópicos, com exceção de algumas poucas disciplinas. Os professores que publicaram ao menos um artigo no IHC-Brasil tendem a explorar os tópicos descritos pelo GT, mas os professores que nunca publicaram no IHC-Brasil exploram menos esses tópicos. Não sabemos as causas desse fato, mas nossa hipótese é de que esses professores, por "não fazerem parte da comunidade", podem nem saber das recomendações para as disciplinas da área. Conjecturamos, que este "problema" pode acontecer com outros tópicos e, portanto, careceria de investigação.

Mesmo sabendo que pode ter ocorrido certa limitação de acesso a materiais didáticos da área, considerando que muitos slides de aulas podem estar em Ambientes Virtuais de Aprendizagem (AVAs) fechados, entendemos que este trabalho conseguiu levantar um rico elenco de materiais que demonstra, de certo modo, o potencial desse tipo de investigação para o Ensino de IHC, que já nos permitiu traçar um panorama preliminar sobre o ensino desses tópicos e seus conteúdos. Entendemos que esse trabalho, portanto, pode contribuir para a melhoria das disciplinas identificadas, além de potencializar que novas experiências didáticas sejam pensadas no Ensino, já que outros pesquisadores-professores de IHC também poderiam aperfeiçoar suas disciplinas.

\subsection{Limitações e ameaças à validade}

Como limitação e ameaças à validade deste trabalho sinalizamos a possibilidade de slides de aulas de docentes que lecionam disciplinas relacionadas a área de IHC não terem sido encontrados, visto que muitos materiais podem estar disponíveis em AVAs de IEs cuja seus conteúdos não estão abertos para o público externo. Outra ameaça a validade do trabalho foi o levantamento dos dados, que foi realizado de forma manual, slide-a-slide, mas que foi mitigado com a checagem dos materiais por ambos os autores. 


\section{Conclusão}

Este artigo apresentou os resultados de um estudo exploratório realizado com o intuito de identificar conteúdos que estão sendo ensinados na área. Resultados deste trabalho indicam que professores participantes da comunidade brasileira de IHC tendem a explorar esses conteúdos em suas disciplinas, mas professores que não são participantes da comunidade exploram menos esses conteúdos, apesar de sua importância para a formação do profissional ou do pesquisador que atuará na área. Como trabalho futuro investigaremos outros conteúdos relacionados ao design e a avaliação de IHC.

\section{Agradecimentos}

Este trabalho foi apoiado pelo Grupo de Pesquisa em Artefatos Físicos de Interação (PAIRG) da Universidade Federal do Rio Grande do Norte (UFRN). Também agradecemos pelos recursos do Laboratório de Computação Física e Fisiológica do PAIRG (PAIRG L2PC) da UFRN.

\section{Referências}

Annett, J. (2003) "Hierarchical Tasks Analysis". In: Hollnagel (ed.) Handbook of Cognitive Task Design.

Bim, S.A., Prates, R.O., Silveira, M.S. e Winckler, M. (2012) "Ensino de IHC Atualizando as Discussões sobre a Experiência Brasileira". In: WEIHC'12.

Card, S., Moran, T. e Newell, A. (1983) "The Psychology of Human-Computer Interaction".

Costa Neto, M.A. e Leite, J.C. (2011) "Empregando Modelo de Interação na Representação de Padrões de Usabilidade”. In: SBSI'11.

Hix, D. e Hartson, H. (1993) "Developing User Interfaces: Ensuring Usability Through Product \& Process".

Leite, J.C. (2010) "Aplicando Técnicas Práticas de Comunicação para o Design e a Avaliação de IHC na Formação de Profissionais de Computação". In: IHC'10.

Lopes, A., Marques, A.B., Conte, T. e Barbosa, S.D.J. (2015) "MoLVERIC: An Inspection Technique for MoLIC Diagrams”. In: SEKE'15.

Muñoz, I.K. (2000) "Validação do Formalismo TAOS para a Modelagem da Tarefa do Usuário no Contexto do Projeto de Interface Homem-Máquina”. Dissertação/UFPB.

Paternó, F., Mancini, C. e Meniconi, S. (1997) “ConcurTaskTrees: A Diagrammatic Notations for Specifying Task Models”. In: INTERACT'97.

Payne, S. e Green, T.R.G. (1989) "Task-action Grammar: The Model and Its Developments". In: Diaper (ed.) Task Analysis for Human-Computer Interaction.

Silveira, M.S. e Prates, R.O. (2007) "Uma Proposta da Comunidade para o Ensino de IHC no Brasil'". In: WEI'07.

Souza, C.S., Leite, J.C., Prates, R.O. e Barbosa, S.D.J. (1999) "Projeto de Interfaces de Usuário: Perspectivas Cognitiva e Semiótica”. In: JAI/CSBC'99.

Winckler, M.A.A. e Pimenta, M.S. (2004) “Análise e Modelagem de Tarefas”. In: IHC'04. 